Supporting Information for

\title{
Facile Electrodeposition of Amorphous Nickel/Nickel Sulfide Composite Films for High-efficiency Hydrogen Evolution Reaction
}

Chengcheng Miao, ${ }^{1}$ Xingwen Zheng, ${ }^{1}$ Jiamin Sun, ${ }^{1}$ Hang Wang, ${ }^{2}$ Jie Qiao, ${ }^{1}$ Ning

Han, ${ }^{2}$ Shanpeng Wang, ${ }^{1}$ Wei Gao, ${ }^{3,}{ }^{*}$ Xiaohui Liu, ${ }^{1}$ and Zai-xing Yang ${ }^{1, *}$

1 School of Physics, School of Microelectronics, State Key Laboratory of Crystal

Materials, Shandong University, Jinan, 250100, P. R. China

2 State Key Laboratory of Multiphase Complex Systems, Institute of Process

Engineering, Chinese Academy of Sciences, Beijing, 100190, P. R. China

3 State Key Laboratory of Solidification Processing, Center for Nano Energy

Materials, School of Materials Science and Engineering, Northwestern

Polytechnical University and Shaanxi Joint Laboratory of Graphene (NPU), Xi'an, 710072, P. R. China 
`E-Mail: wei.gao@nwpu.edu.cn, zaixyang@sdu.edu.cn

\section{Experimental section}

Preparation of Samples. All the chemical reagents are analytical grade and used without any further purification. The electrochemical experiments are performed with a three-electrode system. A piece of cleaned nickel foam with a size of $1.0 \times 1.0 \mathrm{~cm}^{2}$ is used as the working electrode, a graphite rod is employed as the counter electrode, and an $\mathrm{Ag} / \mathrm{AgCl}$ electrode is adopted as the reference electrode. To deposit nickel/nickel sulfide on the $\mathrm{Ni}$ foam, a $75 \mathrm{~mL}$ electrolyte solution containing $0.05 \mathrm{~mol} / \mathrm{L} \mathrm{NiCl}_{2}, 0.10 \mathrm{~mol} / \mathrm{L}$ $\mathrm{CS}\left(\mathrm{NH}_{2}\right)_{2}$, and $0.15 \mathrm{~mol} / \mathrm{L} \mathrm{NH} \mathrm{N}_{4} \mathrm{~F}$ is prepared. The electrodeposition process is carried out with two continuous steps, namely, the first step of a small current density at 0.50 $\mathrm{mA} \cdot \mathrm{cm}^{-2}$ for $90 \mathrm{~min}$ and the second step with a large current density at $10 \mathrm{~mA} \cdot \mathrm{cm}^{-2}$ for 75 min, respectively. The obtained nickel/nickel sulfide composite sample labelled as Sample 1 is washed several times with deionized water, and then dried naturally in air. Meanwhile, the nickel/nickel sulfide composite samples labelled as Sample 2 and Sample 3 are also synthesized by the same method with adding $0.10 \mathrm{~mol} / \mathrm{L}$ and $0.20 \mathrm{~mol} / \mathrm{L}$ $\mathrm{NaH}_{2} \mathrm{PO}_{2} \cdot \mathrm{H}_{2} \mathrm{O}$ into the electrolyte. In addition, Sample 1', Sample 2' and Sample 3' are also prepared on carbon cloth by the same experimental condition with adding $0 \mathrm{~mol} / \mathrm{L}$, $0.10 \mathrm{~mol} / \mathrm{L}$ and $0.20 \mathrm{~mol} / \mathrm{L}$, respectively.

Material Characterization. The phase formation is identified using X-ray diffraction (XRD, D8 Advance, Bruker, $\mathrm{Cu} \mathrm{K} \alpha$ radiation). The composition is tested using 
Inductively Coupled Plasma-Optical Emission Spectrometer (ICP-OES, Agilent 5110). Morphology of the catalysts is observed by a scanning electron microscope (SEM, Nova NanoSEM 450, FEI Company). The X-ray photoelectron spectroscopy (XPS, PHI5000VersaProbe equipped with a monochromatic Al $\mathrm{K} \alpha$ source, ULVAC-PHI) analyses are carried out to investigate the elemental composition and chemical states of the active materials grown on NF. The transmission electron microscopy (TEM) images and selected-area electron diffraction (SAED) patterns are obtained from JEM 2100, JEOL.

Electrochemical Measurements. Electrochemical measurements are performed by an electrochemical workstation on CHI 760E (Shanghai Chenhua Instrument Cvbeo., Ltd.) in a standard three-electrode system. The as-prepared samples on NF are used as working electrodes without further treatments in the alkaline medium of $1.0 \mathrm{M} \mathrm{KOH}$ solution. Graphite electrode and a saturated $\mathrm{Ag} / \mathrm{AgCl}$ electrode are used as counter and reference electrodes, respectively. Linear sweep voltammetry (LSV) is performed at the scan rate of $2 \mathrm{mV} \cdot \mathrm{s}^{-1}$ for HER. The double layer capacitance $\left(\mathrm{C}_{\mathrm{dl}}\right)$ is evaluated by cyclic voltammogram $(\mathrm{CV})$ curves with the scanning rates from 10 to $80 \mathrm{mV} \cdot \mathrm{s}^{-1}$ and from the slope of the plots of capacitive current versus scan rate. Electrochemical impedance spectroscopy (EIS) is carried out at $0 \mathrm{~V}$ vs. reversible hydrogen electrode (RHE) with a frequency range of $10^{-2}$ to $10^{5} \mathrm{~Hz}$ with $\mathrm{AC}$ signal amplitude of $5 \mathrm{mV}$. Equivalent circuit for fitting EIS data is achieved with Zview software. The current density is normalized to 
geometric area of the electrodes. Measured potentials vs. $\mathrm{Ag} / \mathrm{AgCl}$ (saturated solution) are converted to RHE according to the Nernst equation (Eq. 1).

$$
E_{R H E}=E_{A g / A g C l}+0.197+0.059 p H
$$

The stability test of sample is performed by taking $5000 \mathrm{CV}$ cycles at a scan rate of $100 \mathrm{mV} \cdot \mathrm{s}^{-1}$ from -1.0 to $-1.6 \mathrm{~V} \mathrm{vs}$. $\mathrm{Ag} / \mathrm{AgCl}$, and then again LSV is measured with the same condition as the initial one. The stability is also performed by the chronopotentiometry measurements at a fixed current density of $20 \mathrm{~mA} \cdot \mathrm{cm}^{-2}$ for $20 \mathrm{~h}$. 

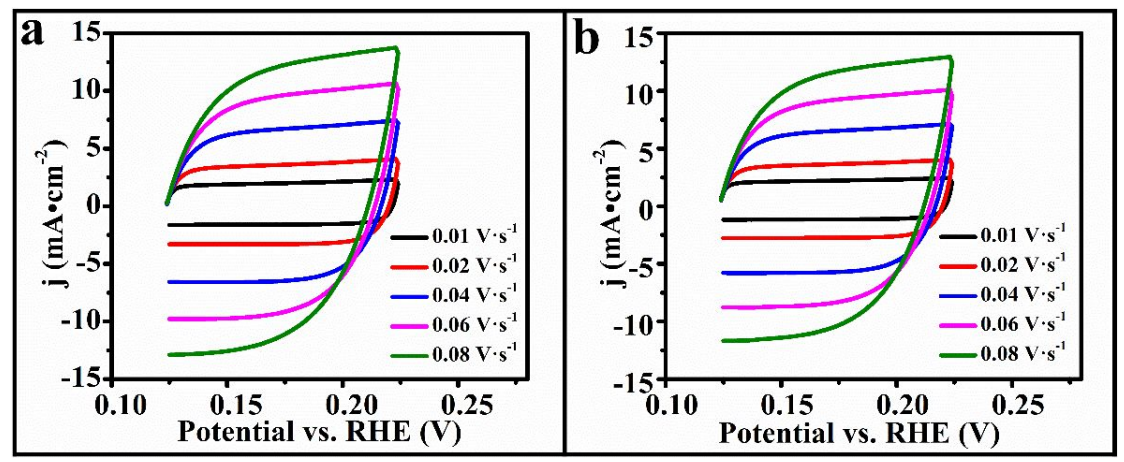

Figure S1. CV plots by different scan rates of prepared samples. (a) Sample 1, (b) Sample 2.

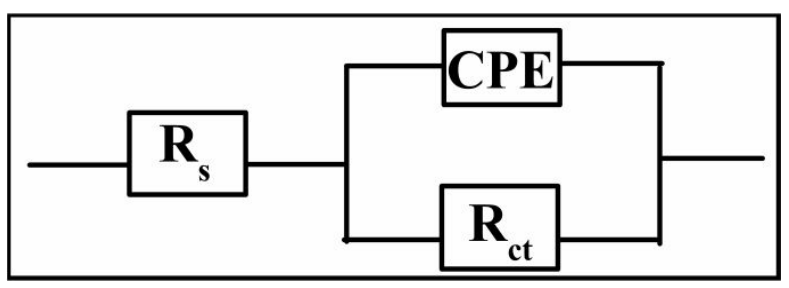

Figure S2. The equivalent circuit of resistances of Nyquist plots.

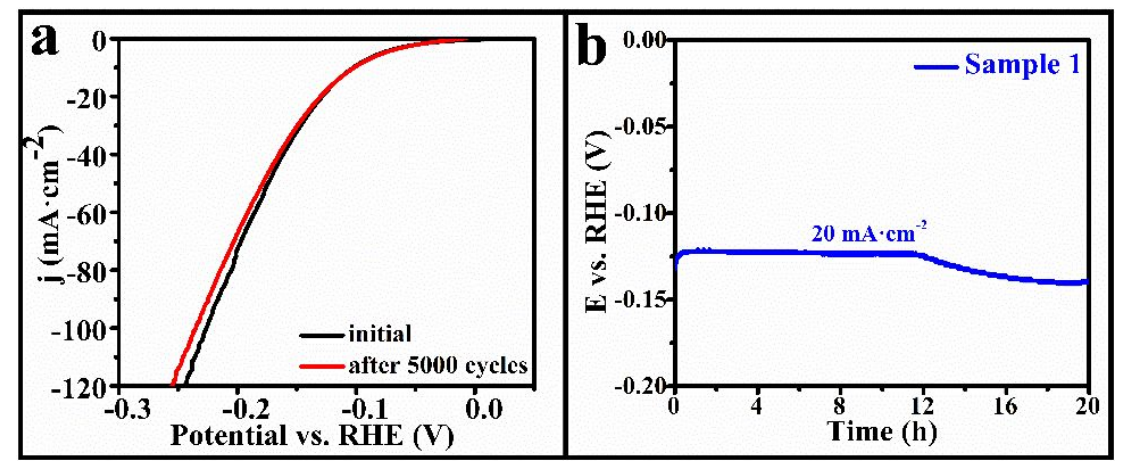

Figure S3. Stability test of Sample 1. (a) linear sweep voltammetry curves of

Sample 1 before and after cyclic voltammogram scanning for 5000 cycles, (b)

Stability test of Sample 2 at $20 \mathrm{~mA} / \mathrm{cm}^{2}$ for $20 \mathrm{~h}$. 


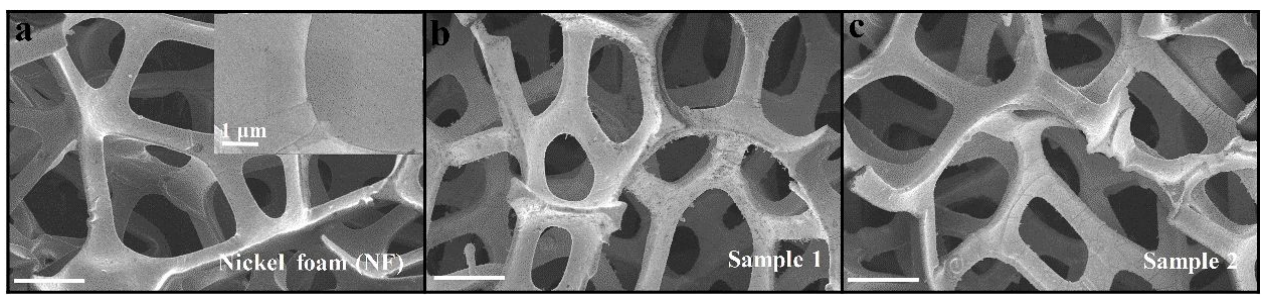

Figure S4. Morphology of samples. SEM images of (a) bare NF, (b) Sample 1, (c) Sample 2. Scale bar is $200 \mu \mathrm{m}$.
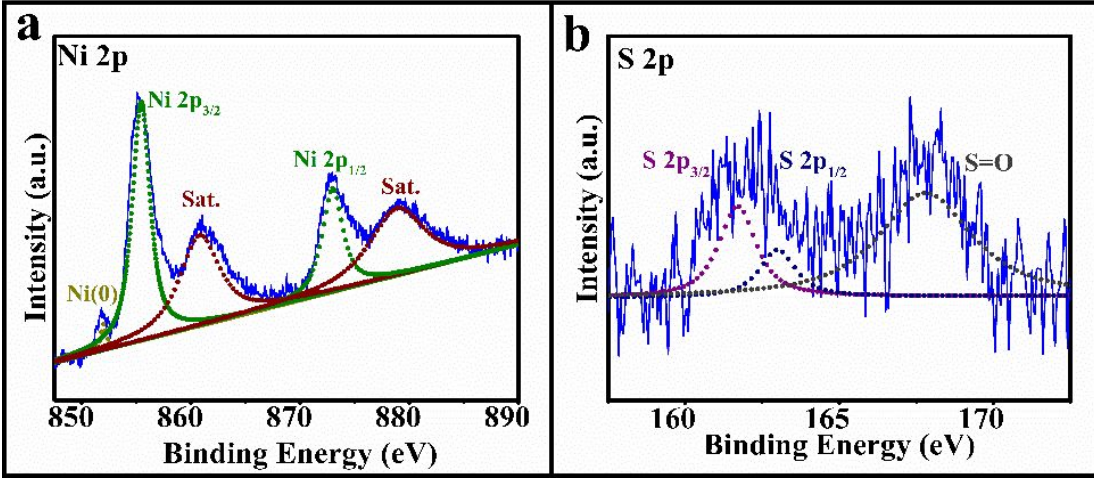

Figure S5. Surface state of Sample 1 after stability test. High-resolution XPS spectra of (a) Ni 2p, (b) S 2p.

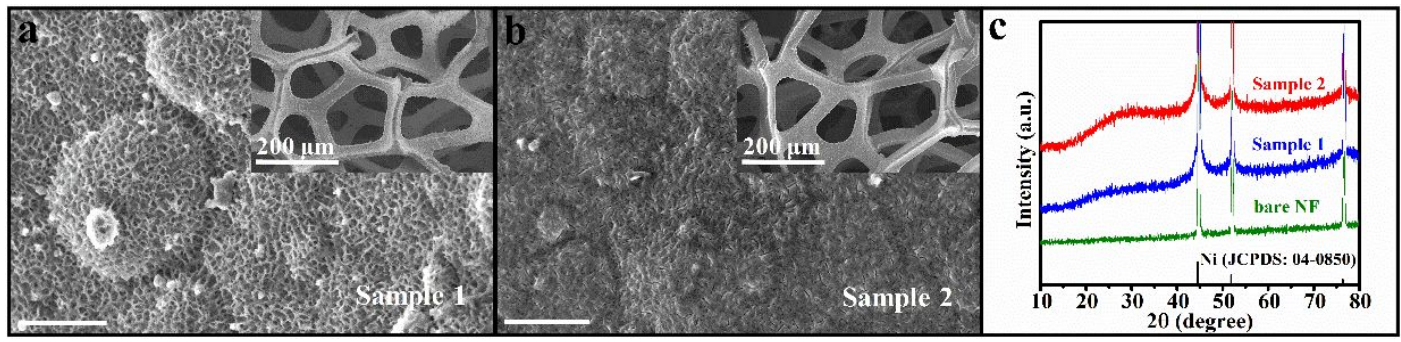

Figure S6. Morphology and crystal structure of samples after stability test. SEM images after stability testing of (a) Sample 1, (b) Sample 2, (c) XRD patterns of all samples after stability testing. Scale bar is $1 \mu \mathrm{m}$. 
Table S1. The molar ratio of as-prepared samples.

\begin{tabular}{cc}
\hline Samples & Ni : S (Molar ratio) \\
\hline Sample 1 & $2.46: 1$ \\
Sample 2 & $3.09: 1$ \\
Sample 3 & $3.60: 1$ \\
\hline
\end{tabular}

